\title{
Performance Evaluation of a Wireless Body Area Sensor Network for Remote Patient Monitoring
}

\author{
Jamil Y. Khan, Mehmet R. Yuce, and Farbood Karami
}

\begin{abstract}
In recent years, interests in the application of Wireless Body Area Network (WBAN) have grown considerably. A WBAN can be used to develop a patient monitoring system which offers flexibility and mobility to patients. Use of a WBAN will also allow the flexibility of setting up a remote monitoring system via either the internet or an intranet. For such medical systems it is very important that a WBAN can collect and transmit data reliably, and in a timely manner to the monitoring entity. In this paper we examine the performance of an IEEE802.15.4/Zigbee MAC based WBAN operating in different patient monitoring environment. We study the performance of a remote patient monitoring system using an OPNET based simulation model.
\end{abstract}

\section{INTRODUCTION}

A Wireless Body Area Network (WBAN) WBAN based on a low cost wireless sensor network technology could greatly benefit patient monitoring systems in hospitals, residential and work environments [1]. A WBAN system allows easy internetworking with other devices and networks, thus offering health care worker easy access to patient's critical and non-critical data. One of the main advantages of a WBAN is to monitor patients remotely using an intranet or the internet. A WBAN could be seen as a special purpose wireless sensor network with a number of additional system design requirements. A WBAN is mostly likely to incorporate wearable and implantable node operating in two different frequencies. An implantable node is most likely to operate at $400 \mathrm{MHz}$ using the MICS medical band whereas the wearable node could operate in ISM/UWB or some other band.

In a patient monitoring system data transmission reliability is extremely important. Considering the importance of the WBAN, IEEE has recently formed a specialist study group. Currently the IEEE802.15.6 group is working on the development of a body area network standard [2]. The new standard will define the PHY (Physical) and MAC (Medium Access Control) layer management issues which could be used to develop a low cost, ultra low power and highly reliable wireless network. These functionalities are controlled primarily by PHY and MAC layers in conjunction with the application layers. The new WBAN standard is likely to be based on the IEEE 802.15.4 MAC layer and a new PHY layer.

A body area network operated in an interconnected manner could offer significant advantage for remote health monitoring. A WBAN can operate in an ad hoc networking mode or in a centralized mode where each sensor nodes transmits its own sensor data to a service node as shown in Fig. 1. Figure shows that multiple sensor nodes will be

\footnotetext{
${ }^{1}$ J. Y. Khan, M. R. Yuce, and F. Karami are with the School of Electrical Eng. and Computer Sci., University of Newcastle, Callaghan, NSW 2308, Australia. Email: Jamil.Khan, Mehmet.Yuce@\{newcastle.edu.au\}
}

transmitting data to a service node which will subsequently transmit the combined data to a record server connected to a hospital network. The service node could be connected via a router or a switch to an internet or to the internet to transmit data to a remote terminal. The intranet or the internet based connection could be a TCP/IP based connection for reliable data transfer. Such a system could easily be developed by integrating WBANs using an existing hospital or medical network. Fig. 1 also shows a remote WBAN network configuration where the WBAN is connected to a hospital computer via a core switch, and a voice and data gateway to the internet. The hospital Zigbee network will be a short range network with a maximum transmission range of 10 meters. In this paper we examine the architecture of this remote patient monitoring network and present some simulation results.

The paper is organized as follows. Section II discusses the remote patient monitoring network based on the WBAN architecture. Section III presents the system architecture and the simulation model. Section IV presents some simulation results, and conclusions and discussions are presented in the section $\mathrm{V}$.

\section{REMOte WBAN ARCHITECTURE}

In this paper we used the IEEE802.15.4/Zigbee based sensor network to implement a WBAN. The Zigbee is a commercial standard which develops the application on top of the IEEE802.15.4 standard that defines the PHY and the MAC layer. It is possible to develop medical applications on a Zigbee standard by appropriately defining higher layer procedures. As mentioned earlier that it is likely that the forthcoming WBAN standard will be based on the IEEE802.15.4 MAC layer hence, the use of above WBAN makes a good candidate for performance evaluation of a remote medical system. In the WBAN all sensor nodes uses the random access protocol known as the CSMA/CA (Carrier Sense Multiple Access/ Collision Avoidance) medium access control protocol to transmit data to the master node. The advantage of a random access protocol is the simplicity of its implementation, lower system cost, and offers a low delay and reliable data transmission. Zigbee devices can transmit up to $250 \mathrm{kbs}$ at $2.4 \mathrm{GHz}$ which is sufficient data rate for typical WBAN applications. Each node will encapsulate its sensor data into an 802.1.5.4 MAC frame and transmit it to the master node.

Table I lists of various physiological signal characteristics and their required data rates. Except the nerve potentials the data rate requirements are very minimal which could be easily handled by Zigbee devices. For remote monitoring purposes each sensor nodes will transmit its packet to the hospital record computer via the service node which operates in a centralized manner. For a remote data access, the hospital computer acts as a buffer to transmit real-time data. The computer aggregates all patient data into a larger TCP block. 


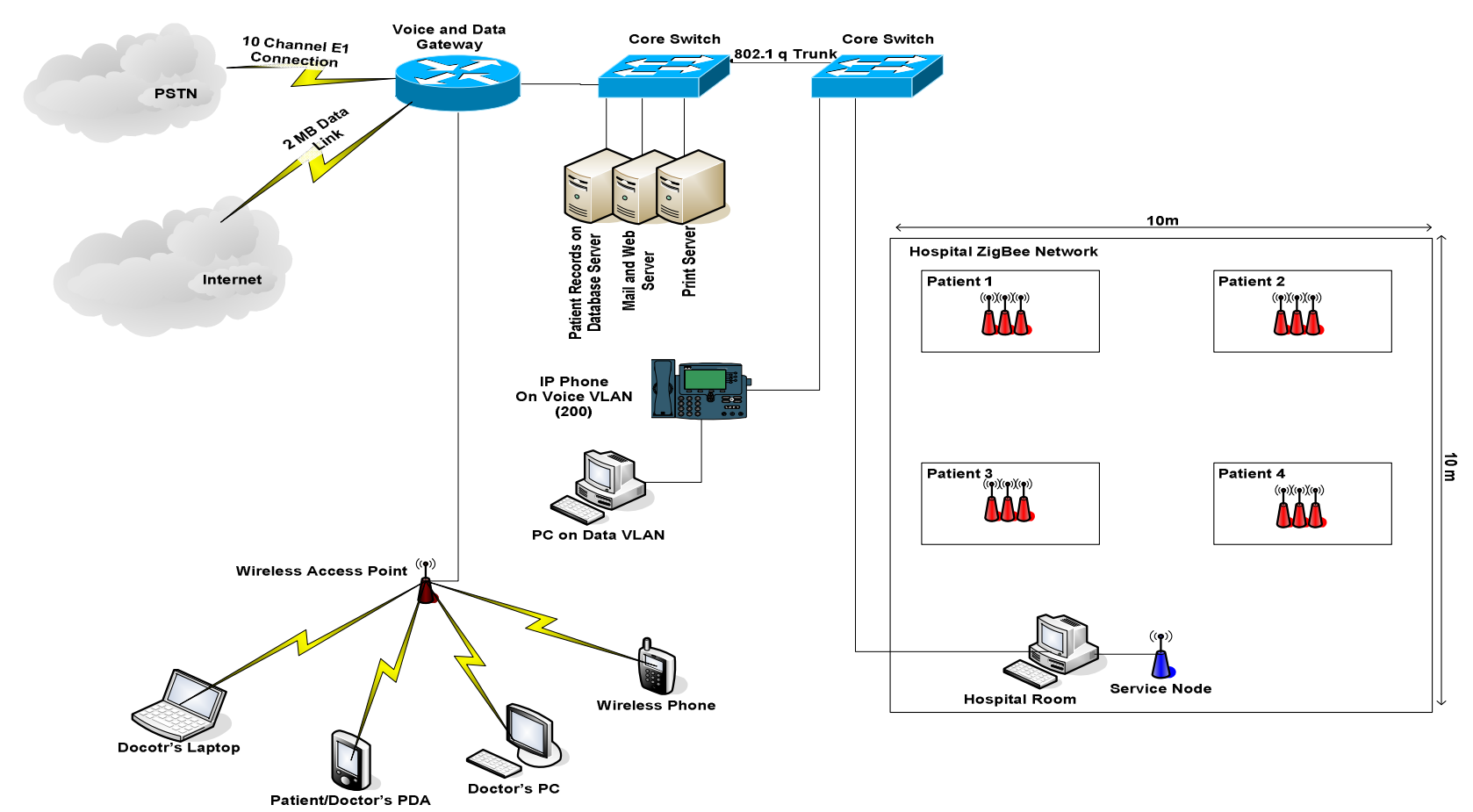

Figure 1: A WBAN operating in a remote patient monitoring environment.

Table: I

\begin{tabular}{|l|l|l|l|l|}
\hline $\begin{array}{l}\text { Physiological } \\
\text { Signal }\end{array}$ & Parameter range & $\begin{array}{l}\text { Data } \\
\text { arrival } \\
\text { time } \\
(\mathrm{sec})\end{array}$ & $\begin{array}{l}\text { Sample } \\
\text { Size } \\
\text { (bits) }\end{array}$ & $\begin{array}{l}\text { Data } \\
\text { rate } \\
(\mathrm{kbs})\end{array}$ \\
\hline Blood flow & $1-300 \mathrm{ml} / \mathrm{s}$ & 0.025 & 12 & 0.48 \\
\hline ECG signal & $0.5-4 \mathrm{mV}$ & 0.002 & 12 & 6.0 \\
\hline $\begin{array}{l}\text { Respiratory } \\
\text { rate }\end{array}$ & $2-50 \mathrm{breaths} / \mathrm{min}$ & 0.05 & 12 & 0.24 \\
\hline Blood Pressure & $10-400 \mathrm{~mm} \mathrm{Hg}$ & 0.01 & 12 & 1.2 \\
\hline Blood pH & $6.8-7.8 \mathrm{pH}$ units & 0.25 & 12 & .048 \\
\hline $\begin{array}{l}\text { Nerve } \\
\text { Potentials }\end{array}$ & $0.01-3 \mathrm{mV}$ & $5 \mathrm{E}-05$ & 12 & 240 \\
\hline $\begin{array}{l}\text { Body } \\
\text { Temperature }\end{array}$ & $32-40^{\circ} \mathrm{C}$ & 5 & 12 & .0024 \\
\hline
\end{tabular}

To design a remote monitoring network, it is necessary to design communication links very efficiently and reliably. Reliability in a medical network can be defined in terms of delay profile, delay jitter and information loss rate. Efficiency of a network can be defined as the ratio of information bits to the total transmitted bits. Reliability of a WBAN will depend on physical and MAC layer procedures as well as on the network topology. The reliability of a remote network which is a fixed network will generally be high; however, occasional packet losses may arise due to congestions and buffer overflow. One of main considerations for a remote medical network will be the TCP/IP (Transmission Control Protocol/Internet Protocol) link.

In our proposed remote network system, any authorized network device whether a mobile or a fixed network device can connect to the patient data via the hospital record computer. In a remote monitoring environment medical data will be transmitted through a network with other types of data. Fig. 1 is an example of such a network used for different applications. In this case it is advisable to use a priority mechanism by setting the appropriate bit(s) of the TOS (Type of Service) field for the IPv4 or the bit(s) of the traffic class field of IPv6 protocol. Another issue requires attention for the remote communication is the packet size design. As shown in Table-1, data samples generated by different sensors are quite small hence; it will be extremely inefficient to transmit these data separately. The hospital computer will aggregate these data into a larger packet to improve the transmission efficiency because the TCP/IP link over an Ethernet network will generate 58 byte packet header. In this case, payload should be sufficiently larger to improve the transmission efficiency.

\section{System ARChitecture AND Simulation}

To analyse the performance of a remote patient monitoring system we used a database on the hospital record computer from where data is transmitted to a remote location. The data base is loaded by the WBAN master node by accepting data from various sensor nodes. Figure 2 shows the medical data obtained via a WBAN and stored on the hospital record computer. As shown in Fig. 2a that it is possible to select patient or particular sensor to retrieve those data remotely via the computer. The WBAN master node periodically uploads data from various sensors. Detail design and performance results of the WBAN can be found in the reference [3]. We simulated a hospital network based on the Ethernet standard. The network connects the database server along with other servers and applications within the hospital network. 


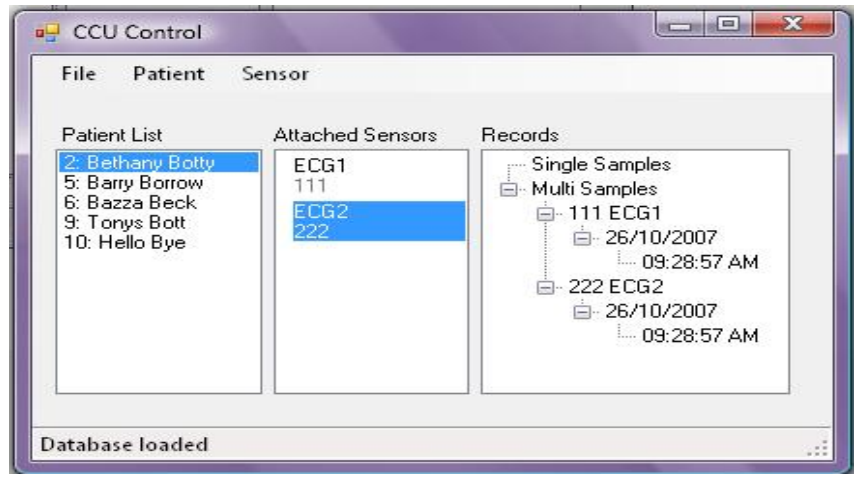

(a)

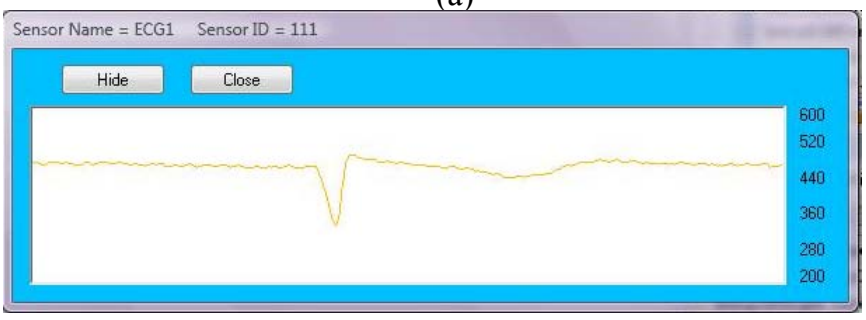

(b)

Figure 2(a): Database upload window (b) retrieved medical record from the database.

A number of network scenarios were simulated using an OPNET model. The model simulated all network components of Fig. 1. Traffic models were developed using Table 1. In the simulation, the database server transmits patient data to remote terminals on a periodic basis. The data base server (hospital computer) sends 4000 bytes per second to the remote computer. The 4000 bytes of data is generated by four patients who are monitored via the WBAN for all physiological signals listed in Table-1 expect the nerve potential. The TCP data block size of 1000 byte is generated by each patient. We use the hospital computer to aggregate all WBAN sensor data to generate a 4000 byte TCP block which is transmitted after every second over the hospital network. In the second simulation we included the nerve potential data transmission along with other physiological data. As seen in the table, the nerve potential sensor generates data at a rate of $240 \mathrm{kbs}$. In this case we generate a TCP block size of 12,000 bytes which is transmitted every $100 \mathrm{~ms}$. To transfer nerve potential data from a patient body, it is necessary to incorporate high data rate WBAN transmitters such as IEEE802.11 standard based wireless local area network [4]. Although the Zigbee standard supports $240 \mathrm{kbs}$ data rate but supporting an application of that data rate will incur long delays. We simulated the hospital network using a $100 \mathrm{Mbs}$ Ethernet network. In the OPNET model we used a CISCO $3500 \mathrm{XL}$ series switch. The simulation model was also loaded with other typical applications such as email, print and web servers were running to generate additional load. Additional loads were put in the model to obtain some realistic simulation results.

\section{Simulation Results}

In this section, we present a number of initial simulation results to analyse the performance of a remote patient monitoring network. We ran an OPNET simulation model for eight simulation hours to obtain performance results. Fig. 3 shows the TCP segment transfer and Ethernet packet delays over the hospital local area network for two different TCP segment generation times. In the first simulation, the service node generates a TCP segment every $0.2 \mathrm{sec}$ with a segment size of 800 bytes by incorporating data from all four patient sensor nodes. In the other simulation TCP segment is generated after every $1 \mathrm{sec}$ with a segment size of 4000 bytes. The total volume of data generated by the service node in both scenarios is same 4000 bytes/sec. Results clearly show that the TCP block delays and Ethernet packet delays are different for two different scenarios. In both simulations the TCP segment size was set equal to the Ethernet packet size. This simulation scenario is for a lightly loaded network where the patient data is transmitted to two remote computers. The total end to end delay from a WBAN will be sum of the Zigbee network transfer delay, packetisation delay and the TCP data block transfer delay. We have simulated the WBAN performance separately. A similar work was reported in another publication which details the WBAN performance by using Zigbee nodes [5]. Typical sensor network to service network packet delay for the Zigbee network is around $25 \mathrm{~ms}$; this delay includes the retransmission delay caused by the channel errors and collisions generated due to the use of the CSMA/CA protocol. Hence, the total one second medical data transfer delay from a WBAN to a remote computer will be $1.031 \mathrm{sec}$, where the packetisation delay is $1 \mathrm{sec}$, WBAN last packet transfer delay is $25 \mathrm{~ms}$ and the TCP data block transfer delay is $6 \mathrm{~ms}$ (average). For the 0.2 packetisation delay the one second medical data transfer delay will be 1.135 sec. Fig. 4 shows the total amount of information received by the remote computer over the TCP connection. Received information volume is the same as the total data generated by four patients.

Next we observed the transmission queue size of the hospital computer which is gathering all sensor node information via the service node. Fig. 5 shows the average queue size distribution. The plot shows that the queue size depends on the TCP segment generation time. Transmission queue size is an important design issue for the record server, particularly if the TCP/IP server is implemented on a microcontroller. A low cost microcontroller based TCP/IP server can easily be implemented on a microcontroller [6].

Finally, we examined the effect of transmitting all physiological data including the nerve potential data in Fig. 6. The average TCP block transfer instantaneous delay which is averaging about $3 \mathrm{~ms}$. In this scenario TCP blocks are transmitted every $100 \mathrm{~ms}$ using the Ethernet size TCP segment. Simulation result shows that the average Ethernet packet delays are similar to previous scenarios but the average TCP block delay is much lower than the second scenario. Lower TCP block delay is attributed to the fact that the frequent arrival of TCP blocks causes lower queuing delay in the TCP queue because the segments are generated at a faster rate. When TCP blocks arrives at a slower rate, the TCP segment generation time increases causing higher endto-end delay. In the simulation we transmitted patient record data as high priority traffic by setting the appropriate field of the IP header which reduces the transmission delay. For example, the print server traffic is transmitted with the lowest 
priority where the average TCP block transmission delay is $20 \mathrm{~ms}$.

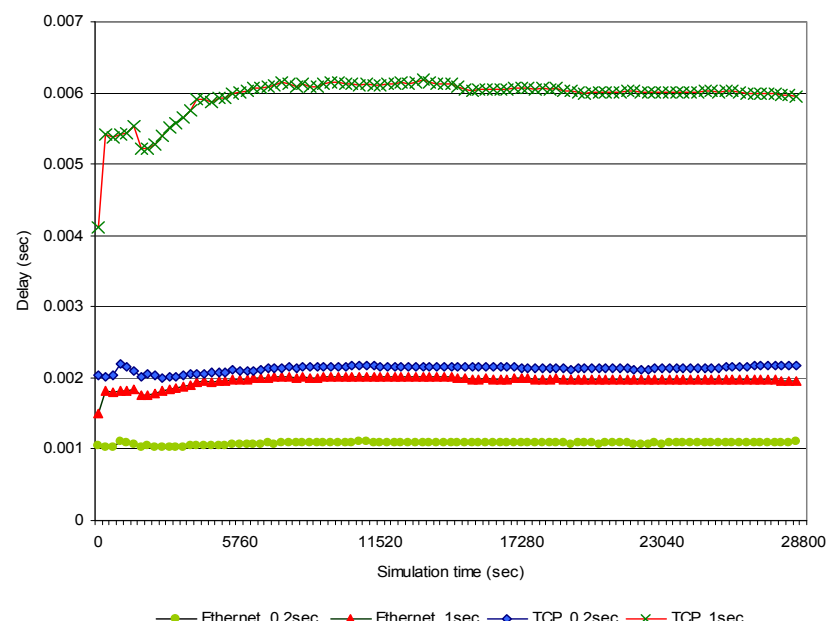

Figure 3: Remote data transfer delay for four monitored patients without the nerve potential data.

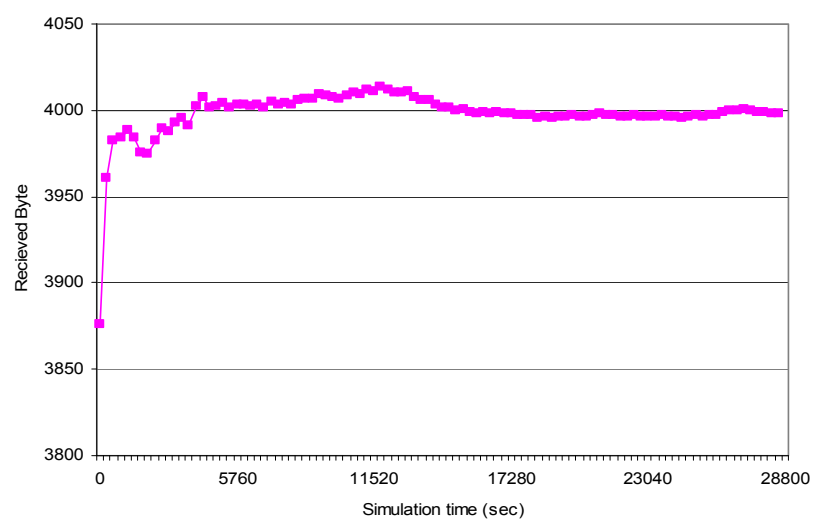

Figure 4: TCP data received by the remote PC generated by 4 patients.

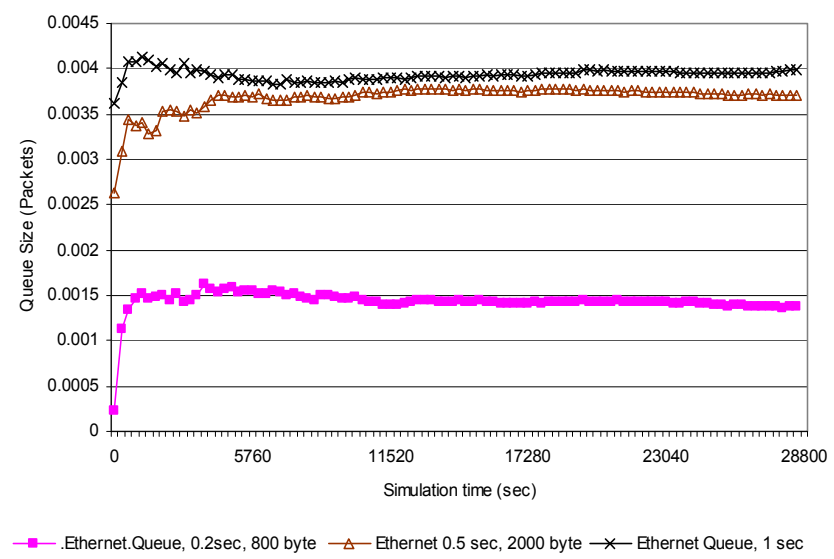

Figure 5: Service node transmission queue size

\section{Discussions ANd CONCLUSIONS}

In this paper we presented the performance results of a remote patient monitoring system using the wireless body sensor network. Simulation results show that it is possible to monitor patients from remote locations using the WBAN over the internet without reasonable delay. For a remote patient monitoring, two design issues are highlighted in this paper. First, a WBAN should not be directly connected to a main hospital network via the service node. With a directly connected WBAN the service node will continuously transmitting short packets which reduce the transmission efficiency of network and increases the transmission delay. As mentioned in the previous section that shorter TCP blocks also increases the end-to-end delay. Table- 1 shows that most of the data packets will be short size even though several data samples might be combined at sensor nodes before transmitting a Zigbee packet. Secondly, the TCP protocol used in a fixed network does not work very well in wireless networks. Hence, it will be sensible to use a multi-hop network i.e. combination of wireless and fixed network links to optimize the transmission time. To implement a remote patient monitoring system we suggest to use a star configuration WBAN where all sensor node sends data to the master (service) node which could combine all sensor data and transmit using a TCP/IP connection for reliable data transmission

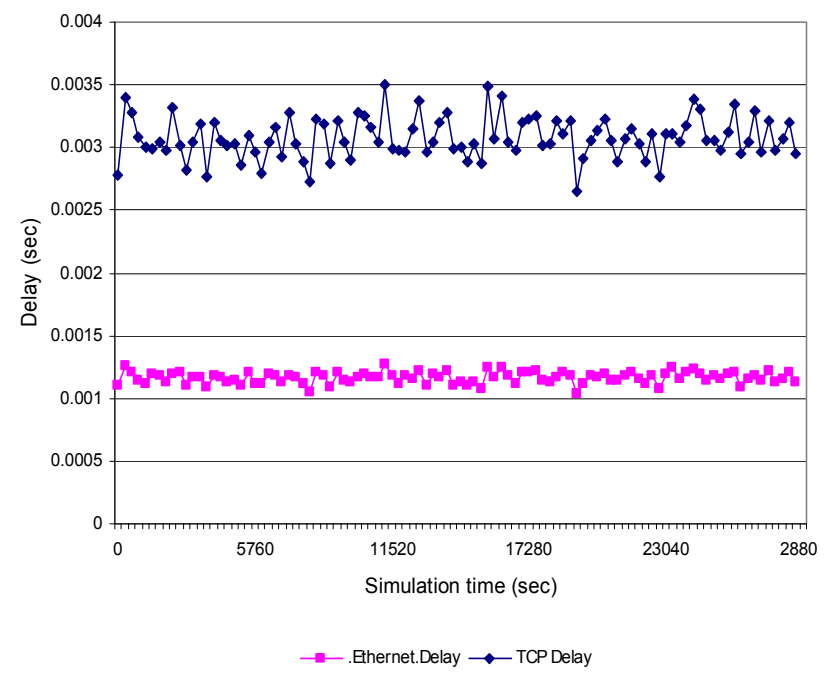

Figure 6: TCP block transmission delay from a single patient including the nerve potential data.

\section{REFERENCES}

[1] C C. Y. Poon and Y-T Zhang, "A Novel Biometrics to Secure Wireless Body Area Sensor Networks for Telemedicine and MHealth", IEEE Communications Magazine, vol:44, no: 4, April 2006, pp. 73-81.

[2] http://www.ieee802.org/15/pub/TG6.html

[3] M. R. Yuce, P. C. Ng, C. K. Lee, J. Y. Khan and Wentai Liu, 'A wireless medical monitoring over a heterogeneous sensor network', 29th Annual International Conference of the IEEE Engineering in Medicine and Biology Society, 2007. Conference Proceedings, Lyon, France (2007).

[4] A. Soomro, D. Cavalcanti, "Opportunities \& Challenges using WPAN and WLAN Technologies in Medical Environments", IEEE Communications Magazine, vol:45, no:2, Feb 2007, page 114-122.

[5] N. Golmie, D. Cypher and O. Rebala, "Performance analysis of low rate wireless technologies for medical applications", Computer Communications, 28(20050, Elsevier, pp. 1266-1275.

[6] http://www.eetimes.eu/semi/206901123 